\title{
Adipose-Derived Stem Cell Transplantation Inhibits Vascular Inflammatory Responses and Endothelial Dysfunction in Rats with Atherosclerosis
}

\author{
Mingqiang Fan ${ }^{1 *}$, Jing Bai ${ }^{1 *}$, Tao Ding ${ }^{1}$, Xiangxiang Yang ${ }^{1}$, Qiaoke $\mathrm{Si}^{1}$, and Dengmei $\mathrm{Nie}^{2}$ \\ ${ }^{1}$ Department of Dardiovascular, Pingliang People's Hospital, Pingliang; \\ ${ }^{2}$ Department of Pathology, Second Provincial People's Hospital, Lanzhou, China.
}

\begin{abstract}
Purpose: This study aimed to investigate the effect of adipose-derived stem cell (ADSC) transplantation on atherosclerosis (AS) and its underlying mechanisms.

Materials and Methods: In our study, rat AS model was established, and ADSCs were isolated and cultured. Atherosclerotic plaque and pathological symptoms of thoracic aorta were measured by Oil Red O staining and Hematoxylin-Eosin staining, respectively. Total cholesterol (TC), triglyceride (TG), high-density lipoprotein cholesterol (HDL-C), and low-density lipoprotein cholesterol (LDL-C) levels were measured by an automatic biochemical analyzer. Expressions of vascular endothelial growth factor (VEGF), vascular cell adhesion molecule-1 (VCAM-1), intercellular adhesion molecule-1 (ICAM-1), aortic endothelin-1 (ET1), interleukin-6 (IL-6), c-reactive protein (CRP), and tumor necrosis factor $\alpha$ (TNF- $\alpha$ ) were measured by enzyme linked immunosorbent assay, VEGF, VCAM-1, ICAM-1, ET-1, respectively, and NF-אB p65 mRNA expressions were detected by quantitative real-

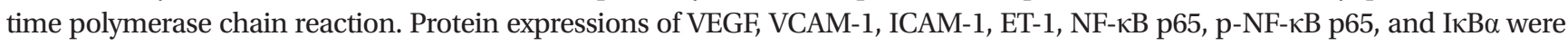
measured by western blot. Moreover, NF- $\mathrm{kB}$ p65 expression was measured by immunofluorescence staining.

Results: ADSC transplantation alleviated the pathological symptoms of aortic AS. ADSC transplantation decreased the levels of TC, TG, and LDL-C and increased serum HDL-C level. Meanwhile, ADSC transplantation decreased the levels of IL-6, CRP, and TNF- $\alpha$ in AS rats. Moreover, the expressions of VEGF, ET-1, VCAM-1, and ICAM-1 were decreased by ADSC transplantation.

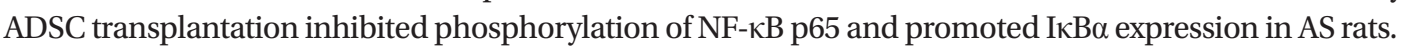

Conclusion: Our study demonstrated that ADSC transplantation could inhibit vascular inflammatory responses and endothelial dysfunction by suppressing NF- $\mathrm{KB}$ pathway in AS rats.
\end{abstract}

Key Words: ADSC, atherosclerosis, endothelial dysfunction, inflammatory responses, NF- $\mathrm{kB}$ pathway

\section{INTRODUCTION}

Atherosclerosis (AS) is a chronic inflammatory disease in arte-

Received: May 14, 2019 Revised: August 12, 2019

Accepted: September 11, 2019

Corresponding author: Dengmei Nie, MD, Department of Pathology, Second Provincial People's Hospital, 1 Hezheng West Road, Chengguan District, Lanzhou, Gansu 730000 , China.

Tel: 86-0933-8639060, Fax: 86-0933-8639060, E-mail: niedengmei@163.com

*Mingqiang Fan and Jing Bai contributed equally to this work.

-The authors have no potential conflicts of interest to disclose.

(C) Copyright: Yonsei University College of Medicine 2019

This is an Open Access article distributed under the terms of the Creative Commons Attribution Non-Commercial License (https://creativecommons.org/licenses/ by-nc/4.0) which permits unrestricted non-commercial use, distribution, and reproduction in any medium, provided the original work is properly cited. rial walls, which is characterized by lipids deposition in the arterial vessel wall. ${ }^{1,2}$ AS causes various acute cardiovascular events, including myocardial infarction and stroke, and severely influences the patients' quality of life. ${ }^{3,4}$ Due to the change in dietary structure, AS incidence has been increasing worldwide and may reach epidemic proportions in the next few decades. ${ }^{5}$ Therefore, it is urgent to find new therapeutic methods and targets for the treatment of AS.

Mesenchymal stem cells (MSCs) have the functions of promoting healing and immunomodulatory, ${ }^{6}$ which provide an opportunity of using autologous MSC transplantation to promote tissue repair and regeneration in clinical practice. ${ }^{7}$ Adipose-derived stem cells (ADSCs) are isolated from adipose tissue, and are ubiquitous in all kinds of MSCs. ${ }^{8}$ Recently, MSC 
transplantation has been recognized as a new technique for treating various diseases. Previously, researchers have reported that ADSCs could enhance liver regeneration in acute liver injury. ${ }^{9}$ Zhang, et al. ${ }^{10}$ have indicated that ADSC transplantation alleviates brain edema from intracerebral hemorrhage. However, precise roles of ADSCs in AS and their potential molecular mechanisms remain to be further investigated.

Nowadays, it is well recognized that endothelial dysfunction is the initial step in the pathogenesis of AS. ${ }^{11}$ The abnormal endothelial function further promotes a series of inflammatory response. ${ }^{12}$ Nuclear factor- $\kappa \mathrm{B}(\mathrm{NF}-\kappa \mathrm{B})$ has long been recognized as a key component of signaling mechanisms involved in the pathogenesis of a number of inflammatory responses. ${ }^{13}$ Under normal physiological condition, NF- $\kappa$ B stays in the cytoplasm by interacting with the inhibitor of $\kappa \mathrm{B}$ (I $\kappa \mathrm{B})$ proteins, of which the prototypical member is I $\mathrm{B} \alpha$. Inflammatory factors, including interleukin-6 (IL-6), c-reactive protein (CRP), and tumor necrosis factor $\alpha$ (TNF- $\alpha)$, could activate $\mathrm{I} \kappa \mathrm{B} \alpha .^{13}$

In our study, we investigated the effects of ADSC transplantation on AS and its related molecular mechanisms. Our results suggested that ADSC transplantation could inhibit vascular inflammatory responses and endothelial dysfunction by suppressing NF- $\kappa \mathrm{B}$ signaling pathway in AS rats. The results of our study may provide new theoretical foundation for deeply exploring the treatment of AS.

\section{MATERIALS AND METHODS}

\section{Animal model of AS}

Forty-eight male Sprague-Dawley (SD) rats (200-220 g) were supplied by Dashuo Co., LTD. (Chengdu, China). The rats were housed at room temperature with $45-60 \%$ humidity for at least a week to adapt to the environment. The rats were randomly assigned to two groups (24 rats in each group): normal group and AS group. Rats in AS group were intraperitoneally injected with vitamin $\mathrm{D}_{3}$ at a dose of $700000 \mathrm{IU} / \mathrm{kg}$ over $3 \mathrm{~d}$. Subsequently, the rats were fed a high-fat vitamin $\mathrm{D}_{3} \operatorname{diet}(2 \%$ cholesterol, $0.5 \%$ sodium cholate, $0.2 \%$ propyl thiouracil, $3 \%$ lard, $5 \%$ sugar, $0.0125 \%$ vitamin $\mathrm{D}_{3}$ powder, and $82.3 \%$ basic diet) every day for 3 weeks. Meanwhile, rats in normal group were fed a normal diet and injected with physiological saline under the same conditions. All animal experiments were conducted strictly in accordance with the guideline for the care and use of laboratory animals, and approved by the ethics committee of Second Provincial People's Hospital.

\section{Determination of serum lipids}

After 3 weeks of modeling, $6 \mathrm{~mL}$ of blood was collected. Total cholesterol (TC), triglyceride (TG), high-density lipoprotein cholesterol (HDL-C), and low-density lipoprotein cholesterol (LDL-C) levels were then measured using an Olympus AU2700 automatic biochemical analyzer (Interscience, Saint Nom La Breteche, France).

\section{Oil Red $O$ staining assay}

The aorta, which was cut from the root to the bifurcation of thoracic arteries, was fixed in $10 \%$ formalin. Aortic roots were sectioned to $5-\mu \mathrm{m}$ thick sections and then stained with Oil Red O for $10 \mathrm{~min}$. Subsequently, the sections were counterstained with hematoxylin to visualize the nuclei. Finally, the plaques were visualized with an optical microscope (Olympus, Tokyo, Japan).

\section{Isolation and culture of ADSCs}

ADSCs were isolated from the subcutaneous adipose tissue of 8-10 week old male C57BL/6J mice. In brief, the subcutaneous adipose tissue was chopped and digested with $0.1 \%$ collagenase type I (Gibco, Grand Island, NY, USA)/1\% BSA solution at $37^{\circ} \mathrm{C}$ for $30 \mathrm{~min}$. Following filtration, centrifugation, and resuspension, ADSCs were cultivated in Dulbecco's Modified Eagle Medium (DMEM) (Gibco, USA) supplemented with $10 \%$ fetal bovine serum (Beckman Coulter Inc., Miami, FL, USA) and $1 \%$ penicillin/streptomycin (Gibco). After $48 \mathrm{~h}$, the adherent cells were washed with phosphate-buffered saline (Sigma-Aldrich Chemie, Steinheim, Germany) to remove loose cellular debris, and the medium was then changed every 3 days. ADSCs at three passage were stained for $15 \mathrm{~min}$ at room temperature with monoclonal PE-conjugated antibodies for CD34 and CD45 (BD Biosciences, San Diego, CA, USA) or FITCconjugated antibodies for CD44 and CD90 (BD Biosciences), and then incubated with the appropriate fluorochrome-conjugated secondary antibody. Isotype control IgG was used to stain the cells as control. Finally, samples were analyzed by flow cytometer (Beckman Coulter, Brea, CA, USA).

\section{ADSC transplantation}

After modeling, normal and AS rats were randomly divided into four groups (eight rats in each group): normal group, normal+ ADSCs group, AS group, and AS+ADSCs group. ADSCs cell suspension, labeled by PKH26 dye (Sigma-Aldrich Chemie) with a concentration of $1 \times 10^{10} \mathrm{~L}^{-1}(0.5 \mathrm{~mL})$, was slowly injected into the rats of normal+ADSCs group and AS+ADSCs group through their tail vein. Furthermore, rats of normal group and AS group were injected with equal volume of DMEM medium.

\section{Enzyme linked immunosorbent assay (ELISA)}

After 3 weeks of ADSC transplantation, the rats were decapitated and their blood was rapidly collected. The levels of vascular endothelial growth factor (VEGF), vascular cell adhesion molecule-1 (VCAM-1), intercellular adhesion molecule-1 (ICAM-1), and aortic endothelin-1 (ET-1) were measured using ELISA kits (R\&D Systems Inc., Minnesota, MN, USA) according to the methods provided by the manufacturer. Meanwhile, inflammatory factors including IL-6, CRP, and TNF- $\alpha$ 
were also detected.

\section{Real-time fluorogenic PCR assay}

Thoracic aortas were collected and the total RNA was extracted using TRIZOL (Invitrogen, Carlsbad, CA, USA) after 3 weeks of ADSCs transplantation. Then, total 500-ng RNA was reverse-transcribed into cDNA by Revert Aid First Strand cDNA Synthesis Kit (Thermo Scientific, Fremont, CA, USA), and measured using quantitative real-time polymerase chain reaction (qRT-PCR) (Bio-Rad Laboratories, Hercules, CA, USA) with SYBR green qPCR Master Mix (Thermo Scientific). Primers used for qRT-PCR analysis were as follows: VEGF (forward): 5'-ATGTGTGTCCGTCTAGATGT-3', (reverse): 5' GGAAGTGTTGATTGGAAAACTGA-3'; ET-1 (forward): 5'-ACCCAGCCTATCCAGAATCC-3', (reverse): 5'-ATGAAGCTGGGCTCTGAGAA-3'; VCAM-1 (forward): 5'GCAGAAGTGGAATTAGTTG-3', (reverse): 5' CTGGGTTCTCCAAGAAAA-3'; ICAM-1 (forward): 5'CACAGGTGGTGCTICTGAAC-3', (reverse): 5'-CTCCTGAGCCTTCTGTAACTTG-3'; GAPDH (forward): 5'-ATTGTCAGCAATGCATCCTG-3', (reverse): 5'-GTAGGCCATGAGGTCCACCA-3'.

\section{Western blot analysis}

Total proteins were extracted from the thoracic aortas by lysis buffer, and protein concentration was measured by BCA kit (TaKaRa, Kyoto, Japan). Protein samples ( $30 \mu \mathrm{g})$ were subjected to $10 \%$ SDS-PAGE and then transferred onto nitrocellulose membrane. Following blocking with 5\% skim milk, the membranes were incubated with the primary antibody (ICAM-1, 1:1000; VCAM-1, 1:1000; ET-1, 1:1000; NF-кB p65, 1:1000; p-NF-кB p65, 1:1000, Abcam, Cambridge, MA, USA; VEGF, 1:1000; IкB $\alpha, 1: 1000$, Cell Signaling Technology, Beverly, MA, USA; GAPDH, 1:1000, Sino Biologial, Beijing, China) at $4^{\circ} \mathrm{C}$ overnight. Afterwards, peroxidase-labeled secondary antibody (anti-rabbit IgG, 1:5000, Abcam) was used for incubation for $1 \mathrm{~h}$ at room temperature. Protein blots were visualized with an enhanced chemiluminescence kit. Finally, density of western blot bands was analyzed using Quantity One 1-D Analysis Software (Bio-Rad Laboratories).

\section{Hematoxylin-Eosin (HE) staining}

The aorta, which was cut from the root to the bifurcation of thoracic arteries, was fixed in $10 \%$ formalin for $24 \mathrm{~h}$. Afterwards, paraffin-embedded tissues were sectioned to 5 - $\mu \mathrm{m}$ thick aorta sections after dehydration and vitrification. The aorta sections were deparaffined with xylene, rehydrated with gradient ethanol, and stained with HE dye in proper order. Histopathological changes were visualized with an optical microscope (Olympus) at $\times 400$ magnification. Moreover, we also measured the thickness of the intima (I) and media of the aorta $(\mathrm{M})$, and calculated the ratio of $\mathrm{I} /(\mathrm{I}+\mathrm{M})$.

\section{Immunofluorescence staining}

Immunofluorescence staining for thoracic aorta tissue was performed on paraffin sections, which were prepared using the same method as in HE staining. After being blocked with $3 \%$ BSA to avoid nonspecific immunoreactions, the sections were incubated overnight at $4^{\circ} \mathrm{C}$ with primary antibody of NF$\kappa B$ p65 (1:100, Abcam). The sections were washed three times and incubated at $37^{\circ} \mathrm{C}$ for $2 \mathrm{~h}$ with FITC-labeled goat anti-rabbit IgG (1:500, Abcam). Afterwards, the sections were stained by 4', 6-diamidino-2-phenylindole (DAPI) for $5 \mathrm{~min}$ at room temperature. Fluorescence images were randomly scanned by a single investigator, who was blind to sample identity, using a confocal laser scanning microscope at $\times 400$ magnification.

\section{Statistical analysis}

All statistical analyses were performed using SPSS ver. 22.0 Statistical Software (IBM Corp., Armonk, NY, USA). The results were presented in the form of mean \pm standard deviation. Differences between various groups were analyzed by one-way ANOVA followed by Tukey's post hoc test, and data of the two groups were assessed using Student's t test. All experiments were repeated three times. $p<0.05$ was considered statistically significant.

\section{RESULTS}

\section{Rats AS model is successfully constructed}

The results of HE staining (Fig. 1A) showed that the aorta in normal group was normal and did not show hyperplasia. However, the aorta had disorganized structures and visible atherosclerotic plaques in AS group. Meanwhile, the intima and media thickness of aorta increased significantly in AS group $(p<0.001)$. The atherosclerotic plaque in aorta was determined by Oil Red O staining (Fig. 1B). Compared to normal group, the number of Oil Red O positive lipid droplets in AS group significantly increased $(p<0.01)$. Furthermore, the ratio of $\mathrm{I} /(\mathrm{I}+\mathrm{M})$ remarkedly increased in AS group compared to normal group $(p<0.001)$ (Fig. 1C). As shown in Fig. 1D, the serum levels of TC, TG, and LDL-C in AS group were significantly higher than those in normal group $(p<0.001)$. In contrast, the serum levels of HDL-C in AS group were significantly lower than those in normal group $(p<0.05)$. All of the results above revealed that AS model of rats was successfully constructed in our research.

\section{Identification of ADSCs}

As shown in Fig. 2A, P3 ADSCs had adherent growth ability, morphology of fibroblast-like cells, and radial shape appearance. The results of flow cytometry confirmed that ADSCs expressed CD44 (98.5\%) and CD90 (97.3\%), but not CD34 (4.12\%) and CD45 (5.41\%), which was consistent with the surface molecular characteristics of ADSCs (Fig. 2B). 

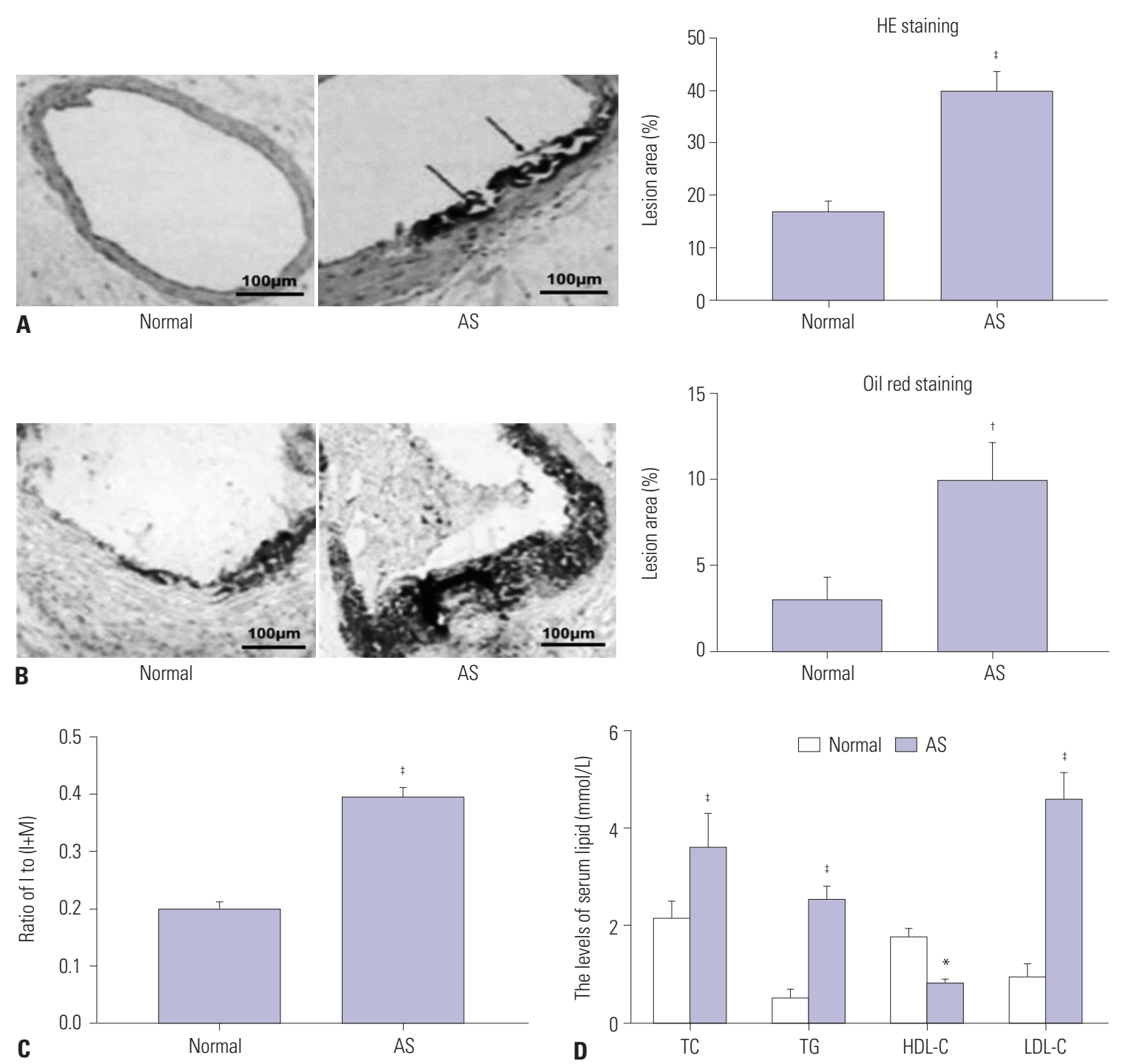

Fig. 1. Successful construction of rat atherosclerosis (AS) model ( $n=8$ in each group). (A) Hematoxylin-Eosin (HE) staining of aortic cross-sections. Arrows indicate atheromatous plaque. Magnification: $\times 200$. (B) Oil Red 0 staining of aortic sections. Magnification: $\times 400$. (C) Ratio of $\mathrm{l} /(\mathrm{I}+\mathrm{M})(\mathrm{I}=$ thickness of aortic intima, M=thickness of aortic media). (D) The levels of total cholesterol (TC), triglyceride (TG), high-density lipoprotein cholesterol (HDL-C), and low-density lipoprotein cholesterol (LDL-C) were measured by automatic biochemical analyzer. Data are presented as mean \pm standard deviation, with repetition of three times. Bar $=100 \mu \mathrm{m}$. ${ }^{*} p<0.05,{ }^{\dagger} p<0.01,{ }^{\ddagger} p<0.001$, vs. Normal group.

\section{ADSCs transplantation alleviates the pathological symptoms of aortic AS in rats}

As shown in Fig. 3A, after 3 weeks of ADSCs transplantation, red positive cells were found in normal+ADSCs group and AS+ADSCs group, while no fluorescent cells were found in normal group and AS group, indicating that ADSC transplantation was successful. In accordance with the aortic morphology observed via HE (Fig. 3B), atherosclerotic plaques were formed in the vascular tissue of AS group $(p<0.001)$, and the thickness of whole vascular wall increased $(p<0.001)$. The visible atherosclerotic plaques and increased vascular wall thickness were alleviated in AS+ADSCs group $(p<0.01)$. Consistent with the results of HE staining, the ratio of $\mathrm{I} /(\mathrm{I}+\mathrm{M})$ significantly increased in AS group compared to normal and normal+ADSCs groups ( $p<0.001)$ (Fig. 3C). Compared to AS group, the ratio of
$\mathrm{I} /(\mathrm{I}+\mathrm{M})$ markedly decreased in AS+ADSCs group $(p<0.01)$ (Fig. $3 C)$. All of these results suggest that ADSC transplantation could alleviate the symptoms of aortic AS.

\section{ADSC transplantation decreases the levels of lood lipids and inflammatory cytokines in AS rats}

Compared to the values in normal and normal+ADSCs group, the serum levels of TC, TG, and LDL-C significantly increased $(p<0.001)$ and serum HDL-C levels markedly decreased in AS group $(p<0.01)$ (Fig. 4A). Compared to AS group, the serum levels of TC, TG, and LDL-C significantly decreased $(p<0.001)$ and serum HDL-C levels markedly increased in AS+ADSCs group $(p<0.05)$ (Fig. 4A). Furthermore, the levels of IL-6, CRP, and TNF- $\alpha$ in AS group significantly increased compared to those in normal and normal+ADSCs groups $(p<0.001)$ (Fig. 4B-D). 

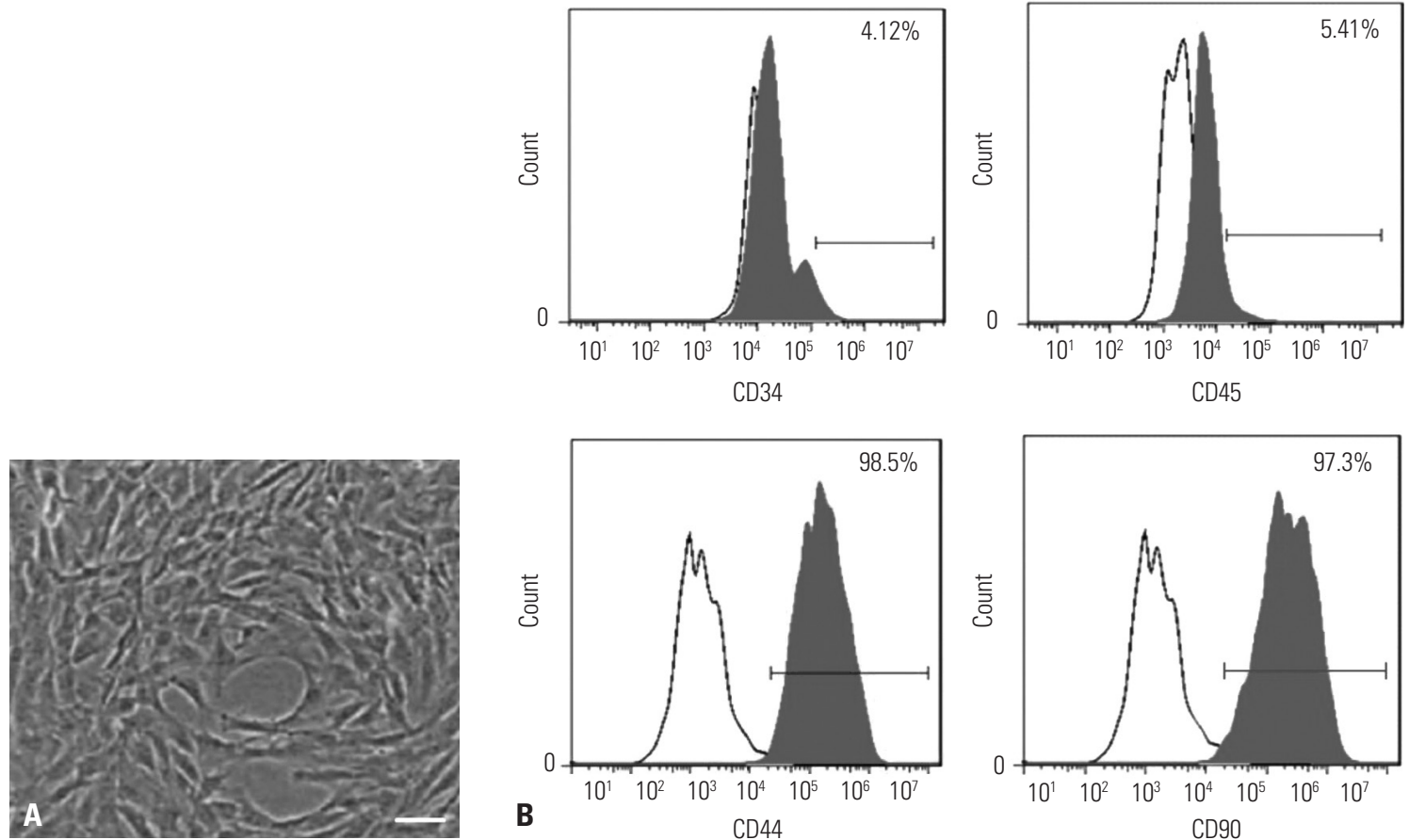

Fig. 2. Identification of adipose-derived stem cells (ADSCs). (A) Morphological characteristics of ADSCs at three passages. Magnification: $\times 200$. Bar $=100 \mu \mathrm{m}$. (B) Expressions of ADSC surface antigens (CD34, CD44, CD45, and CD90) were detected by flow cytometry.

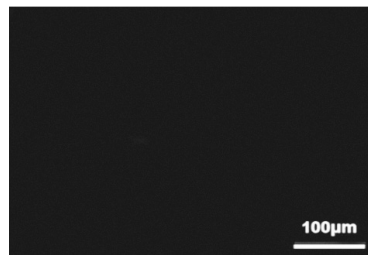

A

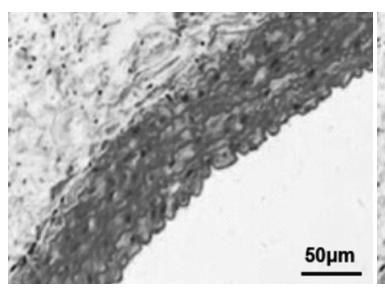

B

Normal

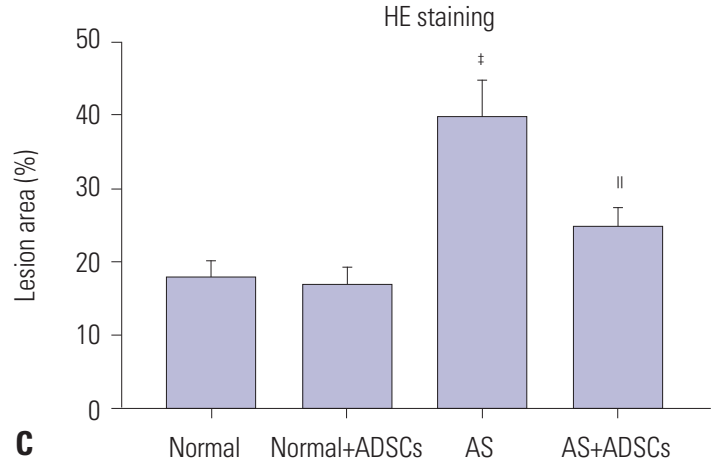

Normal+ADSCs

Normal+ADSCs
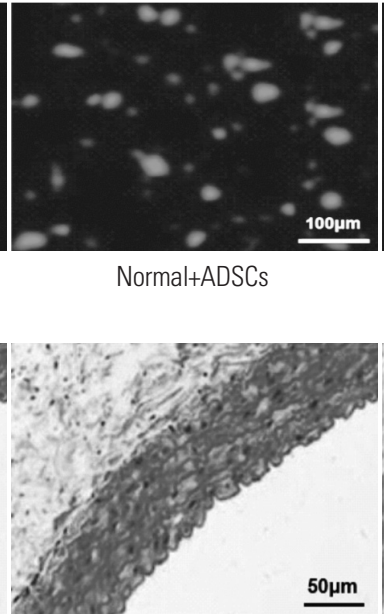

.

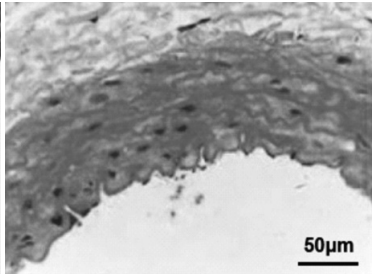

AS

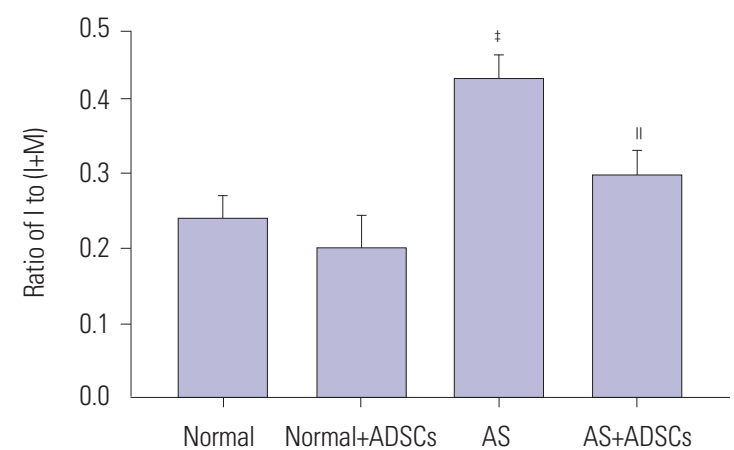

Fig. 3. Adipose-derived stem cells (ADSC) transplantation alleviated the pathological symptoms of aortic atherosclerosis (AS) ( $n=8$ in each group). (A) Expression of PKH26-labeled ADSCs in aortic tissues of rats. Bar $=100 \mu \mathrm{m}$. (B) Hematoxylin-Eosin (HE) staining of aortic sections. Magnification: $\times 200$. (C) Ratio of $\mathrm{I} /(\mathrm{I}+\mathrm{M})$ (I=thickness of aortic intima, $\mathrm{M}=$ thickness of aortic media). Data are presented as mean \pm standard deviation, with repetition of three times. ${ }^{\ddagger} p<0.001$, vs. Normal group and Normal+ADSCs group; " $p<0.01$, vs. AS group. 
The levels of IL-6 $(p<0.001), \mathrm{CRP}(p<0.001)$, and TNF- $\alpha(p<0.01)$ in AS+ADSCs group were lower than those in AS group (Fig. $4 \mathrm{~B}-\mathrm{D})$.
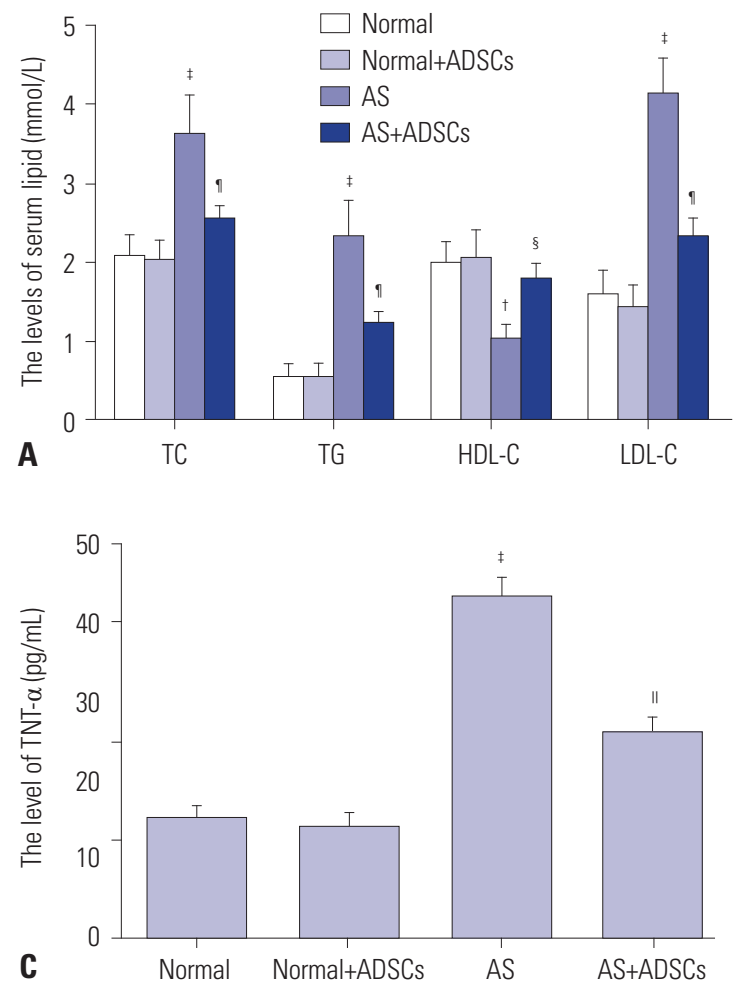

\section{ADSCs transplantation inhibits endothelial dysfunction in AS rats}

The results of ELISA revealed that the levels of VEGF, ET-1,
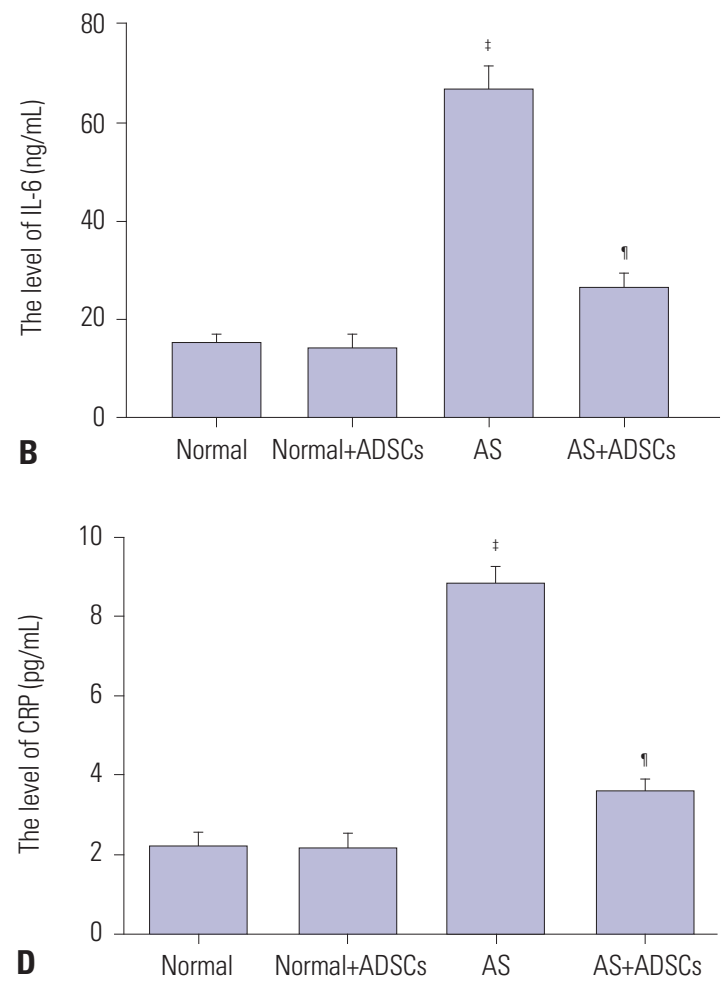

Fig. 4. Adipose-derived stem cell (ADSC) transplantation decreased the levels of lood lipids and inflammatory cytokines in atherosclerosis (AS) rats ( $n=8$ in each group). (A) The levels of total cholesterol (TC), triglyceride (TG), high-density lipoprotein cholesterol (HDL-C), and low-density lipoprotein cholesterol (LDL-C) were measured by automatic biochemical analyzer. (B-D) Expression of interleukin-6 (IL-6), tumor necrosis factor $\alpha$ (TNF- $\alpha$ ), and c-reactive protein (CRP) was detected by Enzyme linked immunosorbent assay. Data are presented as mean \pm standard deviation, with repetition of three times. ${ }^{\dagger} p<0.01,{ }^{\ddagger} p<0.001$, vs. Normal group and Normal+ADSCs group; ${ }^{\S} p<0.05, " p<0.01,{ }^{\natural} p<0.001$, vs. AS group.
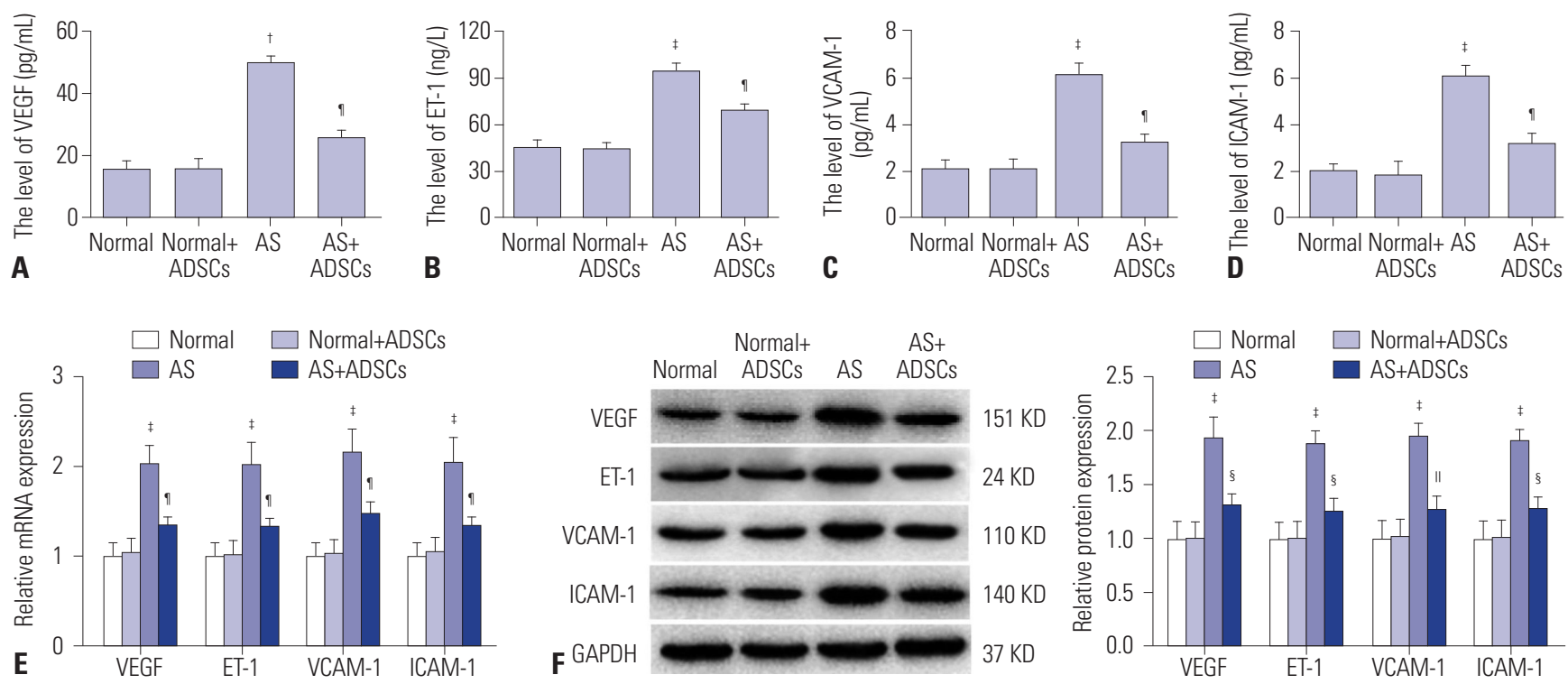

Fig. 5. Adipose-derived stem cell (ADSC) transplantation inhibited endothelial dysfunction in atherosclerosis (AS) rats ( $n=8$ in each group). (A-D) The levels of vascular endothelial growth factor (VEGF), endothelin-1 (ET-1), vascular cell adhesion molecule-1 (VCAM-1), and intercellular adhesion molecule-1 (ICAM-1) were detected by Enzyme linked immunosorbent assay. (E) The mRNA expressions of VEGF, ET-1, VCAM-1, and ICAM-1 were measured by quantitative real-time polymerase chain reaction (qRT-PCR). (F) Protein expressions of VEGF, ET-1, VCAM-1, and ICAM-1 were measured by western blot. Data are presented as mean \pm standard deviation, with repetition of three times. ${ }^{\dagger} p<0.01,{ }^{\ddagger} p<0.001$, vs. Normal group and Normal+ADSCs group; ${ }^{\S} p<0.05, " p<0.01,{ }^{n} p<0.001$, vs. AS group. GAPDH, glyceraldehyde 3-phosphate dehydrogenase. 


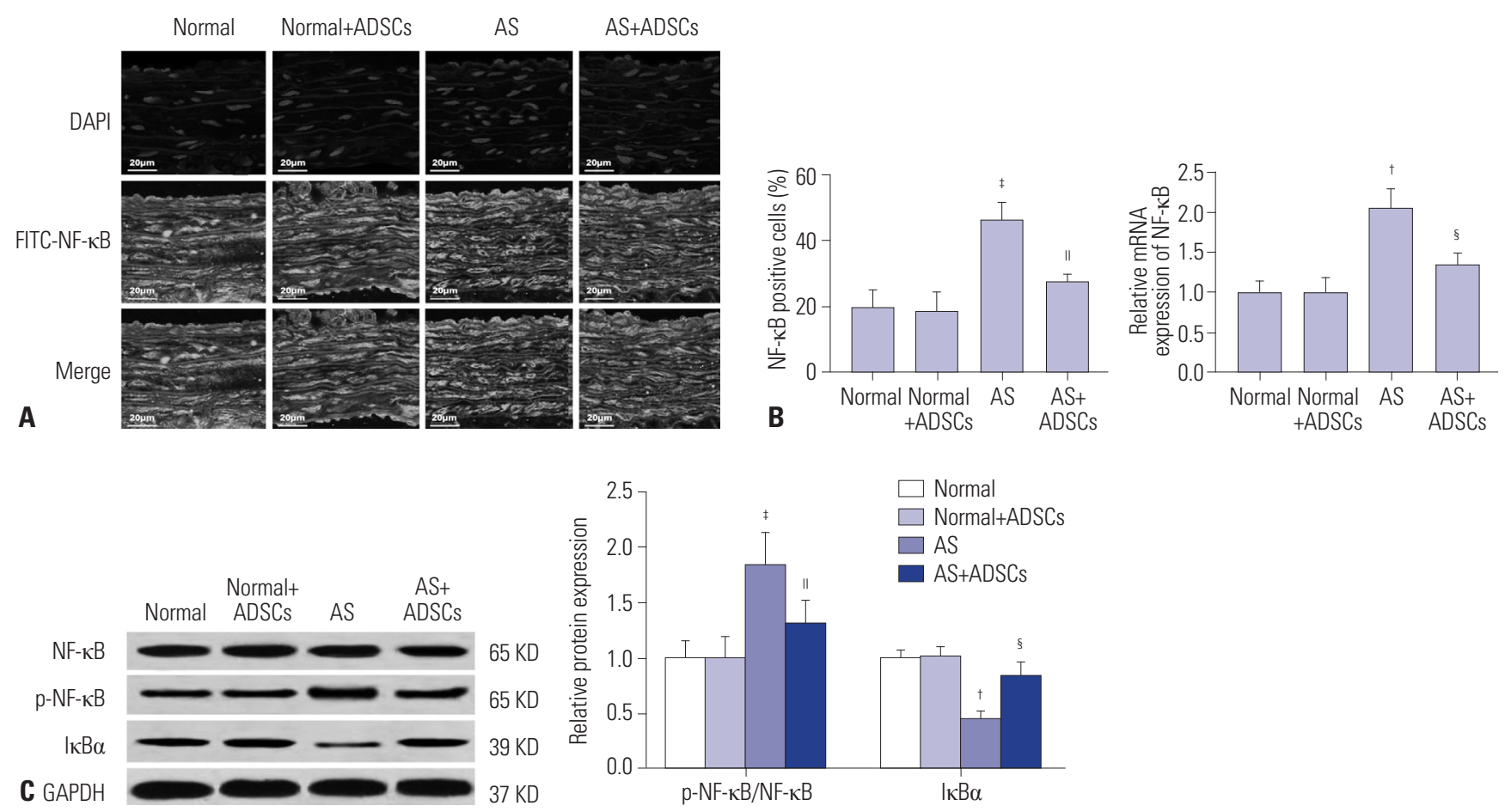

Fig. 6. Adipose-derived stem cell (ADSC) transplantation inhibited NF- $\kappa B$ signaling pathway in atherosclerosis (AS) rats $(n=8$ in each group). (A) Expression of NF- $\kappa B$ p 65 was measured by immunofluorescence staining. Scale bar $=20 \mu \mathrm{m}$. (B) Expression of NF- $\kappa B$ p 65 was detected by quantitative real-time polymerase chain reaction (qRT-PCR). (C) Expressions of NF- $\kappa B$ p65, $p-N F-\kappa B$ p65, and I $\kappa B \alpha$ were measured by western blot. Data are presented as mean \pm standard deviation, with repetition of three times. ${ }^{\dagger} p<0.01,{ }^{\ddagger} p<0.001$, vs. Normal group and Normal+ADSCs group; ${ }^{\S} p<0.05,{ }^{\|} p<0.01$, vs. AS group. DAPI, 4', 6-diamidino-2-phenylindole; GAPDH, glyceraldehyde 3-phosphate dehydrogenase.

VCAM-1, and ICAM-1 in AS group were remarkedly higher than those in normal and normal+ADSCs groups $(p<0.001)$ (Fig. 5A-D). Compared to AS group, the levels of VEGF, ET-1, VCAM-1, and ICAM-1 decreased in AS+ADSCs group $(p<0.001)$ (Fig. 5A-D). Meanwhile, the mRNA and protein expressions of VEGF, ET-1, VCAM-1, and ICAM-1 were measured by qRTPCR (Fig. 5E) and western blot (Fig. 5F). The results also confirmed that both mRNA and protein expressions of VEGF, ET1, VCAM-1, and ICAM-1 in AS group significantly increased compared to those in normal and normal+ADSCs groups $(p<$ 0.001 ). The mRNA expressions of VEGF, ET-1, VCAM-1, and ICAM-1 in AS+ADSCs group dramatically decreased compared to AS group $(p<0.001)$. Similarly, the protein expressions of VEGF $(p<0.05)$, ET-1 ( $p<0.05)$, VCAM-1 $(p<0.01)$, and ICAM$1(p<0.05)$ in AS+ADSCs group were significantly lower than those in AS group. All of these results suggest that ADSC transplantation could inhibit endothelial dysfunction in AS rats.

\section{ADSCs transplantation inhibits NF- $\kappa$ B signaling pathway in AS rats}

$\mathrm{NF}-\kappa \mathrm{B}$ is essential in regulating inflammatory gene expression. Activation of NF- $\kappa \mathrm{B}$ was presented as the ratio of NF- $\kappa \mathrm{B}$ p65 in cytoplasmic to nuclear localization. To confirm the activation of NF- $\mathrm{KB}$ in thoracic aortas, immunofluorescence staining was performed to detect the translocation of NF- $\kappa \mathrm{B}$ p65 in four different groups (Fig. 6A). Immunofluorescence staining results showed that the number of NF- $\kappa \mathrm{B}$ p65-positive cells markedly increased in AS group compared to normal and normal+ADSCs groups ( $p<0.001)$. Compared to AS group, the number of NF- $\kappa \mathrm{B}$ p65-positive cells decreased in AS+ADSCs group $(p<0.01)$. The results were also confirmed by qRT-PCR (Fig. 6B). The mRNA expression of NF- $\kappa \mathrm{B}$ p65 markedly increased in AS group compared to normal and normal+ADSCs groups $(p<0.01)$. Compared to AS group, the mRNA expression of NF- $\kappa \mathrm{B}$ p65 decreased in AS+ADSCs group ( $p<0.05)$. In addition, western blot results revealed that p-NF- $\kappa \mathrm{B}$ p65 expression in AS group significantly increased compared to normal and normal+ADSCs groups $(p<0.001)$ (Fig. 6C). However, the expression of $\mathrm{I} \kappa \mathrm{B} \alpha$ in AS group was markedly lower compared to normal and normal+ADSCs groups $(p<0.01)$ (Fig. $6 \mathrm{C})$. Compared to AS group, $\mathrm{p}-\mathrm{NF}-\kappa \mathrm{B}$ p65 expression decreased $(p<0.01)$ and $\mathrm{I} \kappa \mathrm{B} \alpha$ expression increased $(p<0.05)$ in AS+ADSCs group (Fig. 6C). All of these results suggest that ADSC transplantation could inhibit NF- $\kappa$ B signaling pathway in AS rats.

\section{DISCUSSION}

AS is a chronic disease of the arterial wall, which is the main reason for death and loss of life expectancy of patients all over the world. ${ }^{14,15}$ Therefore, it is urgent to explore and discover in- 
novative and effective treatment strategies to better treat this disease. In the present study, we demonstrated that ADSC transplantation could inhibit vascular inflammatory responses and endothelial dysfunction by suppressing NF- $\mathrm{kB}$ signaling pathway in AS rats.

MSCs could be isolated from various tissues including bones, embryos, fat, muscles, and bone marrow. ${ }^{16}$ Previous research have confirmed that MSCs play important roles in tissue healing and regeneration. ${ }^{17,18} \mathrm{ADSCs}$, which are a group of multipotent stem cells isolated from adipose tissue, are ubiquitous and can be easily harvested. ${ }^{19}$ Recently, transplantation of MSCs was shown to improve the recovery of patients in various diseases. ${ }^{20}$ Therefore, ADSCs could be considered as ideal seed cells in regenerative medicine therapies and translational medicine research. Previously, researchers have reported that ADSCs could enhance liver regeneration in acute liver injury. ${ }^{9}$ In our study, we investigated the effects of ADSC transplantation in AS, and found that ADSCs transplantation could alleviate the pathological symptoms of aortic AS. Moreover, some studies have demonstrated that the levels of TC and LDL$\mathrm{C}$ are higher in AS, while HDL-C level is lower. ${ }^{21}$ Our results confirmed that ADSC transplantation could decrease the serum levels of TC, TG, and LDL-C and increase HDL-C level, suggesting that ADSC transplantation could be beneficial for the treatment of AS.

Accumulating evidence have demonstrated that endothelial dysfunction is the initial step in the pathogenesis of AS. ${ }^{11}$ Endothelial dysfunction is an imbalance of vascular regulator produced by vascular endothelium, such as nitric oxide and ET- $1 .{ }^{22}$ It is worth noting that ET-1 is an indicator molecules in the formation of $\mathrm{AS}^{23}$ which also controls the effects of vasoconstrictors. ${ }^{24}$ Adhesion molecules (ICAM- 1 and VCAM- 1 ) released by vascular endothelium are considered as other activators of early AS. ${ }^{25}$ VEGF is a chemotactic factor during vascular inflammation, which is correlated to the progression of AS. ${ }^{26,27}$ In the present study, we found that ADSC transplantation could decrease the expressions of VEGF, ET-1, VCAM-1, and ICAM1 in AS rats. Subsequently, the abnormal endothelial function further promotes a series of inflammatory responses. ${ }^{12}$ Some research have shown that inflammatory factors could promote the development of AS. ${ }^{28}$ Our results have confirmed that ADSC transplantation could decrease the levels of IL-6, CRP, and TNF- $\alpha$ in AS rats. NF- $\kappa B$ is a pleiotropic regulator of numerous genes involved in innate immunity and inflammatory response, and it also regulates the secretion of inflammatory mediators, such TNF- $\alpha$ IL-6, and IL- $1 \beta .^{29}$ In the canonical NF$\kappa \mathrm{B}$ signaling pathway, the phosphorylation and degradation

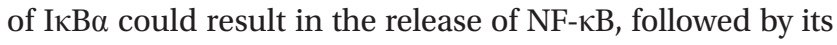
translocation to the nucleus where it regulates proinflammatory gene expression. ${ }^{30,31}$ Accumulating evidence has demonstrated that NF- $\kappa B$ activation is an crucial pathway in the development of AS..$^{32}$ Furthermore, NF- $\mathrm{kB}$ signaling pathway contributes to the upregulation of ICAM- 1 and VCAM- $1 .{ }^{33}$
Zhou, et al. ${ }^{34}$ have reported that human ADSCs play an important role in immunoregulation by inhibiting NF- $\mathrm{kB}$ activation in T cells via PD-L1/PD-1 and Gal-9/TIM-3 pathways. In the present study, we investigated whether ADSC transplantation could play a role in AS through NF- $\mathrm{kB}$ signaling pathway, and found that ADSC transplantation could decrease the phosphorylation of NF- $\kappa B$ p65 and increase the expression of I $\kappa B \alpha$. Our results suggested that ADSC transplantation could inhibit NF- $\kappa B$ signaling pathway in AS rats.

In conclusion, ADSC transplantation could inhibit vascular inflammatory responses and endothelial dysfunction through the inhibition of NF- $\mathrm{kB}$ signaling pathway in AS rats. Our research provides an innovatively regulatory mechanism regarding ADSC transplantation in AS, and suggests a new way to treat AS. Further studies are needed to identify the mechanisms by which ADSC transplantation regulate other inflammatory signaling pathways, including mitogen-activated protein kinase family pathways and extracellular signal-regulated kinase.

\section{AUTHOR CONTRIBUTIONS}

Conceptualization: Mingqiang Fan. Data curation: Jing Bai. Formal analysis: Mingqiang Fan and Tao Ding. Investigation: Mingqiang Fan. Methodology: Xiangxiang Yang. Project administration: Mingqiang Fan. Resources: Qiaoke Si. Software: Qiaoke Si. Supervision: Dengmei Nie. Validation: Dengmei Nie. Visualization: Dengmei Nie. Writingoriginal draft: All authors. Writing—review \& editing: All authors.

\section{ORCID iDs}

$\begin{array}{ll}\text { Mingqiang Fan } & \text { https://orcid.org/0000-0002-3795-054X } \\ \text { Jing Bai } & \text { https://orcid.org/0000-0003-2629-4097 } \\ \text { Tao Ding } & \text { https://orcid.org/0000-0002-7042-375X } \\ \text { Xiangxiang Yang } & \text { https://orcid.org/0000-0002-0974-5952 } \\ \text { Qiaoke Si } & \text { https://orcid.org/0000-0002-4295-0915 } \\ \text { Dengmei Nie } & \text { https://orcid.org/0000-0001-9091-6052 }\end{array}$

\section{REFERENCES}

1. Emini Veseli B, Perrotta P, De Meyer GRA, Roth L, Van der Donckt C, Martinet W, et al. Animal models of atherosclerosis. Eur J Pharmacol 2017;816:3-13.

2. Ross R. Atherosclerosis is an inflammatory disease. Am Heart J 1999;138(5 Pt 2):S419-20.

3. Libby P, Ridker PM, Hansson GK. Progress and challenges in translating the biology of atherosclerosis. Nature 2011;473:317-25.

4. Weber C, Noels H. Atherosclerosis: current pathogenesis and therapeutic options. Nat Med 2011;17:1410-22.

5. Hansson GK. Inflammation, atherosclerosis, and coronary artery disease. N Engl J Med 2005;352:1685-95.

6. Schlosser S, Dennler C, Schweizer R, Eberli D, Stein JV, Enzmann V, et al. Paracrine effects of mesenchymal stem cells enhance vascular regeneration in ischemic murine skin. Microvasc Res 2012;83: 267-75.

7. Mimeault M, Hauke R, Batra SK. Stem cells: a revolution in therapeutics-recent advances in stem cell biology and their therapeu- 
tic applications in regenerative medicine and cancer therapies. Clin Pharmacol Ther 2007;82:252-64.

8. Zuk PA, Zhu M, Ashjian P, De Ugarte DA, Huang JI, Mizuno H, et al. Human adipose tissue is a source of multipotent stem cells. Mol Biol Cell 2002;13:4279-95.

9. Choi JS, Ryu HA, Cheon SH, Kim SW. Human adipose derived stem cells exhibit enhanced liver regeneration in acute liver injury by controlled releasing hepatocyte growth factor. Cell Physiol Biochem 2019;52:935-50.

10. Zhang Y, Deng H, Hu Y, Pan C, Wu G, Li Q, et al. Adipose-derived mesenchymal stem cells stereotactic transplantation alleviate brain edema from intracerebral hemorrhage. J Cell Biochem 2019;120:14372-82.

11. Onat D, Brillon D, Colombo PC, Schmidt AM. Human vascular endothelial cells: a model system for studying vascular inflammation in diabetes and atherosclerosis. Curr Diab Rep 2011;11:193202.

12. Libby P. Inflammation in atherosclerosis. Arterioscler Thromb Vasc Biol 2012;32:2045-51.

13. Negi G, Sharma SS. Inhibition of IкB kinase (IKK) protects against peripheral nerve dysfunction of experimental diabetes. Mol Neurobiol 2015;51:591-8.

14. Charo IF, Taub R. Anti-inflammatory therapeutics for the treatment of atherosclerosis. Nat Rev Drug Discov 2011;10:365-76.

15. Taleb S. Inflammation in atherosclerosis. Arch Cardiovasc Dis 2016;109:708-15.

16. Baranowska A, Skowron B, Nowak B, Ciesielczyk K, Guzdek P, Gil $\mathrm{K}$, et al. Changes in viability of rat adipose-derived stem cells isolated from abdominal/perinuclear adipose tissue stimulated with pulsed electromagnetic field. J Physiol Pharmacol 2017;68:253-64.

17. Maziarz A, Kocan B, Bester M, Budzik S, Cholewa M, Ochiya T, et al. How electromagnetic fields can influence adult stem cells: positive and negative impacts. Stem Cell Res Ther 2016;7:54.

18. Pittenger MF, Mackay AM, Beck SC, Jaiswal RK, Douglas R, Mosca JD, et al. Multilineage potential of adult human mesenchymal stem cells. Science 1999;284:143-7.

19. Liu MH, Li Y, Han L, Zhang YY, Wang D, Wang ZH, et al. Adiposederived stem cells were impaired in restricting CD4(+)T cell proliferation and polarization in type 2 diabetic ApoE(-/-) mouse. Mol Immunol 2017;87:152-60.

20. Lee JS, Hong JM, Moon GJ, Lee PH, Ahn YH, Bang OY, et al. A longterm follow-up study of intravenous autologous mesenchymal stem cell transplantation in patients with ischemic stroke. Stem Cells 2010;28:1099-106.

21. Skrzep-Poloczek B, Tomasik A, Tarnawski R, Hyla-Klekot L, Dyduch A, Wojciechowska C, et al. Nephrotic origin hyperlipidemia, relative reduction of vitamin $\mathrm{E}$ level and subsequent oxidative stress may promote atherosclerosis. Nephron 2001;89:68-72.

22. Giannotti G, Landmesser U. Endothelial dysfunction as an early sign of atherosclerosis. Herz 2007;32:568-72.

23. Fang Y, Sang H, Yuan N, Sun H, Yao S, Wang J, et al. Ethanolic extract of propolis inhibits atherosclerosis in ApoE-knockout mice. Lipids Health Dis 2013;12:123.

24. Hou HF, Yuan N, Guo Q, Sun T, Li C, Liu JB, et al. Citreoviridin enhances atherogenesis in hypercholesterolemic ApoE-deficient mice via upregulating inflammation and endothelial dysfunction. PLoS One 2015;10:e0125956.

25. Krieglstein CF, Granger DN. Adhesion molecules and their role in vascular disease. Am J Hypertens 2001;14(6 Pt 2):44S-54S.

26. Kim I, Moon SO, Kim SH, Kim HJ, Koh YS, Koh GY. Vascular endothelial growth factor expression of intercellular adhesion molecule 1 (ICAM-1), vascular cell adhesion molecule 1 (VCAM-1), and E-selectin through nuclear factor-kappa B activation in endothelial cells. J Biol Chem 2001;276:7614-20.

27. Wang HW, Lo HH, Chiu YL, Chang SJ, Huang PH, Liao KH, et al. Dysregulated miR-361-5p/VEGF axis in the plasma and endothelial progenitor cells of patients with coronary artery disease. PLoS One 2014;9:e98070.

28. Alexander MR, Moehle CW, Johnson JL, Yang Z, Lee JK, Jackson CL, et al. Genetic inactivation of IL-1 signaling enhances atherosclerotic plaque instability and reduces outward vessel remodeling in advanced atherosclerosis in mice. J Clin Invest 2012;122:70-9.

29. Gao Y, Jiang W, Dong C, Li C, Fu X, Min L, et al. Anti-inflammatory effects of sophocarpine in LPS-induced RAW 264.7 cells via NF- $\mathrm{KB}$ and MAPKs signaling pathways. Toxicol In Vitro 2012;26:1-6.

30. Israf DA, Khaizurin TA, Syahida A, Lajis NH, Khozirah S. Cardamonin inhibits COX and iNOS expression via inhibition of p65NFkappaB nuclear translocation and Ikappa-B phosphorylation in RAW 264.7 macrophage cells. Mol Immunol 2007;44:673-9.

31. Pateras I, Giaginis C, Tsigris C, Patsouris E, Theocharis S. NF- $\mathrm{BB}$ signaling at the crossroads of inflammation and atherogenesis: searching for new therapeutic links. Expert Opin Ther Targets 2014; 18:1089-101.

32. Brånén L, Hovgaard L, Nitulescu M, Bengtsson E, Nilsson J, Jovinge S. Inhibition of tumor necrosis factor-alpha reduces atherosclerosis in apolipoprotein E knockout mice. Arterioscler Thromb Vasc Biol 2004;24:2137-42.

33. Cao C, Zhu Y, Chen W, Li L, Qi Y, Wang X, et al. IKK $\alpha$ knockout prevents high fat diet induced arterial atherosclerosis and NF- $\mathrm{kB}$ signaling in mice. PLoS One 2013;8:e64930.

34. Zhou K, Guo S, Tong S, Sun Q, Li F, Zhang X, et al. Immunosuppression of human adipose-derived stem cells on $\mathrm{T}$ cell subsets via the reduction of NF-kappaB activation mediated by PD-L1/PD-1 and Gal-9/TIM-3 pathways. Stem Cells Dev 2018;27:1191-202. 